\title{
Resting Energy Expenditure in Normal-Weight and Overweight/Obese Subjects Was Similar Despite Elevated Sympathovagal Balance
}

\author{
Ugur Dal ${ }^{a} \quad$ A.Taner Erdogan ${ }^{b} \quad$ Asligul Cureoglu $^{a}$ Huseyin Beydagi ${ }^{a}$ \\ aDepartment of Physiology, Medical Faculty, b School of Physical Education and Sports, \\ Mersin University, Mersin, Turkey
}

\section{Key Words}

Autonomic nervous system • Energy expenditure - Fat-free mass · Heart rate variability • Obesity

\begin{abstract}
Objective: Alterations in the autonomic nervous system (ANS) may cause impairment in the metabolic processes that can lead to weight gain. The purpose of this study was to determine the difference between the resting energy expenditure (REE) and the resting ANS activity in overweight/obese and normal-weight healthy subjects. Method: Group 1 consisted of 18 subjects with $\mathrm{BMI}>25 \mathrm{~kg} / \mathrm{m}^{2}$, and 20 subjects with BMI ranging from 20 to $25 \mathrm{~kg} / \mathrm{m}^{2}$ formed group 2. Measurements of low-frequency (LF) and high-frequency (HF) power components expressed in normalized units (LFnu, HFnu) and LF/HF ratio were assessed for analysis of heart rate variability, and simultaneously REE measurement was performed. Results: The mean LFnu (27.2\% increased) and the LF/HF were higher and the mean HFnu was lower (29.9\% decreased) in group 1 than in group $2(p<0.01)$. Although a statistical difference was observed in REE between groups, REE per kilogram corrected for fat-free mass (REE $E_{F F M c o r r}$ ) was $21.47 \pm 2.92$ $\mathrm{kcal} /$ day $/ \mathrm{kg}$ in group 1 , and $21.56 \pm 1.90 \mathrm{kcal} /$ day $/ \mathrm{kg}$ in group 2, and this difference was not statistically significant ( $p>0.05$ ). Conclusion: We concluded that REE $\mathrm{FFMcorr}_{\text {in }}$ overweight/ obese and normal-weight subjects were similar despite elevated sympathovagal balance in

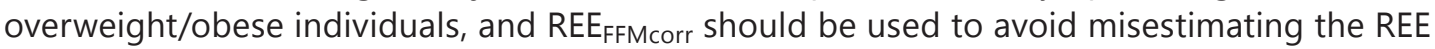
in obese and normal-weight subjects.

Copyright (c) 2012 S. Karger GmbH, Freiburg
\end{abstract}


Dal et al.: Resting Energy Expenditure in Normal-Weight and Overweight/Obese

Subjects Was Similar Despite Elevated Sympathovagal Balance

\section{Introduction}

Obesity and overweight are the major risk factors for metabolic and cardiovascular diseases, and their prevalence is increasing throughout the world [1]. Eating disorders and sedentary lifestyle are the main reasons for gaining weight, but in some cases genetically mediated metabolic properties or disorders can be the cause of obesity [2-4]. The autonomic nervous system (ANS) plays a vital role in the metabolic processes of homeostatic regulation in the body. The alterations in the ANS result in impairment in the metabolic processes that can lead to weight gain [5].

The term heart rate variability (HRV) refers to variations in both heart rate and RR intervals [6]. Sinoatrial node, which determines the normal rhythm of the heart, is regulated by innervations from both the sympathetic and parasympathetic components of the ANS [7]. HRV analysis is used for the assessment of autonomic cardiac activity that may enable the evaluation of the central regulation and interaction of sympathetic and parasympathetic system activity [5-7]. Cardiac sympathetic and parasympathetic activities, highfrequency (HF), low-frequency (LF) components of $\mathrm{HRV}$, and their ratio can be determined by power spectral analysis of HRV [8].

Obesity may alter the balance between the sympathetic and parasympathetic nervous system [9]. There is no consensus about the relationship between obesity and ANS activity level. The opposite hypothesis that most obese subjects are low in sympathetic activity (the MONA LISA hypothesis) [10] was also confirmed by some studies $[9,11]$.

Determination of the energy expenditure by indirect calorimetry is a gold standard method in the clinical practice [12]. The resting energy expenditure (REE) measurement has been used in the obesity-related research to assess the balance between energy intake and energy expenditure [13]. Estimation of energy expenditure is also required in designing a weight loss program. Exploring the relationship between REE and ANS in obese subjects may have clinical impact in in optimizing weight loss and maintenance programs, and especially for pharmacological interventions aiming at a stimulation of the sympathetic nervous system (SNS) activity.

This study was designed to determine whether or not there is a difference between REE and the resting ANS activity in overweight/obese and normal weight healthy subjects.

\section{Material and Methods}

Subjects

16 female and 22 male Caucasian normotensive healthy volunteers between the ages of 17-45 years (mean age $=29.24 \pm 7.16$ years; mean height $=169.32 \pm 10.48 \mathrm{~cm}$; mean mass $=74.34 \pm 18.07$ $\mathrm{kg}$ ) were recruited for the study. All participants were healthy non-smokers and were free of physical illness which can be detected by a physical examination. Their weights were stable for 6 months $( \pm 2$ $\mathrm{kg}$ ), and none of them was on a diet to lose weight. The subjects were not regularly taking any medications, and subjects taking medications that might have influenced HRV and REE were excluded from the study. Participants were classified in two groups based on their BMI. Group 1 consisted of 18 subjects with BMI $>25 \mathrm{~kg} / \mathrm{m}^{2}$, and 20 subjects with BMI ranging from 20 to $25 \mathrm{~kg} / \mathrm{m}^{2}$ formed group 2 . Ethical approval was taken from the local ethical committee, and an informed consent was also obtained from all participants. All procedures used in this study were in agreement with the Declaration of Helsinki.

\section{Anthropometric Measurements}

Weight and height were measured with subjects wearing light clothing and shoes removed. BMI was calculated as weight in kilograms divided by height in meters squared $\left(\mathrm{kg} / \mathrm{m}^{2}\right)$. Subcutaneous fat thickness was measured using a skinfold caliper (Holtain ${ }^{\circledR}$ LTD, Crymych, UK) by the same trained technician at 
three pre-defined locations (triceps, abdominal/suprailiac and thigh). The assessment of the body composition by using skinfold caliper is proven to be consistent with bioelectric impedance analysis [14].

Body density was estimated from the equation of Jackson and Pollack and the Siri's Formula was used to estimate the percentage body fat from body density $[15,16]$. Fat-free mass (FFM) was determined as the difference between body mass and fat mass, where fat mass equaled body mass times percent body fat. FFM was corrected by the normalization method (FFMcorr) described in details by Ravussin and Bogardus [17].

\section{Experimental Procedures}

Subjects were instructed not to consume any food or beverages except water for $12 \mathrm{~h}$ before the test and not to engage in any type of physical exercise on the test day [18]. HRV and REE measurements were performed simultaneously between 8:00 and 09:30 a.m. in a semidarkened and quiet laboratory room specially prepared for REE measurements. Temperature and relative humidity were set to $20-24{ }^{\circ} \mathrm{C}$ and $50 \%$, respectively by using an air-conditioning system.

Subjects rested for $10 \mathrm{~min}$ in a comfortable supine position with spontaneous breathing before the initiation of HRV and REE measurements. Then, HRV and REE were determined simultaneously while the subject lay face up for $20 \mathrm{~min}$.

As it is known, breathing frequencies modify the influence of autonomic system on sinoatrial node [7]. In order to detect the effect of wearing a mask for metabolic measurements on the breathing frequencies of the participants, we recorded the breathing frequencies of the subjects for 1 min by visual observation at the 5 th and 10 th min of resting period, and it was compared with the breathing frequencies taken at the 10 th and 20th min of REE measurements while subjects wearing a mask.

\section{Metabolic Measurements}

The REE measurement was assessed via the indirect calorimetry method (Vmax Spectra ${ }^{\circledR} 29 \mathrm{c}$, Yorba Linda, CA, USA). The gas analyzer was calibrated prior to each test to certified calibration gases $\left(26 \% \mathrm{O}_{2}\right.$ and $74 \% \mathrm{~N}_{2}, 16 \% \mathrm{O}_{2}$ and $4 \% \mathrm{CO}_{2}$ ) according to the manufacturer's recommendations. Using a $3 \mathrm{~L}$ syringe, the mass flow sensor was also calibrated before each experimental trial. The gas samples were collected breath by breath for 20 min on the supine position. The REE recordings were visually inspected, and the smoothest 5-min interval data in steady state condition were chosen to be used in the calculation of REE using the Abbreviated Weir Formula [19]. HRV was also obtained from the same time interval. All subjects were instructed not to move, talk or sleep during the REE measurement. The subjects were warned verbally in case of falling into sleep. Energy expenditure was determined during the follicular phase for all women to discard the influence of hormonal changes in the menstrual cycle during the measurement of REE [20]. REE (kcal/day) and REE which was adjusted for FFM (kcal/day/kg) were analyzed to assess the differences in metabolic rate between groups.

\section{HRV Measurements}

RR intervals were measured using the Polar Heart Rate Monitor (S810, Polar ${ }^{\circledR}$ Electo Oy, Kempele, Finland) for $20 \mathrm{~min}$ in supine position at a sampling frequency of $1000 \mathrm{~Hz}$. The validity of the Polar S810 has been evidenced by studies of Gamelin et al. [21], Vanderlei et al. [22], and Nunan et al. [23]. The Polar S810 wrist receiver and chest strap were placed according to the manufacturer's recommendations.

\section{HRV Analysis}

HRV recordings derived from the POLAR S810 were transferred to a personal computer using the Polar Precision Performance 4.03 software. The raw HRV data were edited by the Polar Precision software (moderate filter power, minimum protection zone $6 \mathrm{bpm}$ ). The HRV record was discarded when the amount of abnormal beats was more than 2\% [24]. Following artifact correction, RR intervals were exported under American Standard Code for Information Interchange (ASCII) format [21] to be analyzed with Kubois ${ }^{\circledR}$ HRV analysis Software v2 (Biosignal Laboratory, University of Kuopio, Kuopio, Finland) [25]. HRV analyses were performed in accordance with The Task Force of the European Society of Cardiology and the North American Society of Pacing and Electrophysiology recommendations [6]. A segment of $256 \mathrm{~s}$ was selected within the $300 \mathrm{~s}$ of the supine recording corresponding to the time of 5 min smoothest REE data to compute the mean RR interval [21].The same segment used in the time domain analysis was resampled at $4 \mathrm{~Hz}$ and detrended for subsequent analysis. An autoregressive model was used to determine the power spectrum densities of the HRV analysis. The power spectrum for the frequency domain HRV analysis was 


\begin{tabular}{l|l}
\hline \multicolumn{2}{l}{ Obes Facts 2012;5:776-783 } \\
\hline DOI: 10.1159/000345189 & $\begin{array}{l}\text { (c) 2012 S. Karger GmbH, Freiburg } \\
\text { www.karger.com/ofa }\end{array}$ \\
\hline Published online: October 26, 2012 &
\end{tabular}

Dal et al.: Resting Energy Expenditure in Normal-Weight and Overweight/Obese

Subjects Was Similar Despite Elevated Sympathovagal Balance

Table 1. Demographic anthropometric data of the groups ${ }^{\mathrm{a}}$

\begin{tabular}{|c|c|c|}
\hline Variables & $\begin{array}{l}\text { Group 1 } \\
(\mathrm{n}=18)\end{array}$ & $\begin{array}{l}\text { Group } 2 \\
(\mathrm{n}=20)\end{array}$ \\
\hline Age, years & $31.06 \pm 7.73$ & $27.60 \pm 6.36$ \\
\hline Height, cm & $171.67 \pm 12.14$ & $167.20 \pm 8.49$ \\
\hline Weight, kg & $87.28 \pm 17.37^{*}$ & $62.70 \pm 8.05$ \\
\hline BMI, $\mathrm{kg} / \mathrm{m}^{2}$ & $29.47 \pm 3.87^{*}$ & $22.36 \pm 1.54$ \\
\hline Body fat, \% & $29.02 \pm 6.94^{*}$ & $19.84 \pm 7.26$ \\
\hline Fat mass, $\mathrm{kg}$ & $25.46 \pm 9.45^{*}$ & $12.32 \pm 4.68$ \\
\hline FFMcorr, kg & $74.55 \pm 12.50^{*}$ & $63.12 \pm 8.62$ \\
\hline
\end{tabular}

divided into three bands: very low frequency (VLF 0.0033-0.04 Hz), LF (0.04-0.15 Hz) and HF (0.15-0.40 $\mathrm{Hz}$ ). Measurements of LF and HF power components expressed in normalized units (LFnu, HFnu), LF/HF ratio, and total power were assessed for statistical analysis [6].

\section{Statistical Analysis}

All statistical analyses were performed using SPSS for Windows v11.5.1 (SPSS ${ }^{\circledR}$ Inc. Chicago, IL, USA). The Shapiro-Wilk normality test was used to assess the normal distribution of the continuous variables separately for each group. According to normality test, all demographic and anthropometric data, except weight, were normally distributed in both groups. A log transformation was applied to total power and $\mathrm{LF} / \mathrm{HF}$ that was not distributed normally. The Mann-Whitney nonparametric $t$ test was used to assess the difference between groups in REE and FFMcorr. The independent $t$ test was used to evaluate significant differences between groups for normally distributed variables. Chi-square test was used to assess gender distribution between groups. The relationship between REE and FFMcorr was assessed by linear regression test. Graph was drawn by use of GraphPad Prism Ver. 3.0 (GraphPad ${ }^{\circledR}$ Software Inc., San Diego, CA, USA).

\section{Results}

The subjects were divided into two groups according to their BMI. In group 1, there were 11 men and 7 women (10 subjects with BMI between 25.1 and $29.9 \mathrm{~kg} / \mathrm{m}^{2}, 8$ subjects with BMI $\geq 30 \mathrm{~kg} / \mathrm{m}^{2}$ ). Group 2 consisted of 11 men and 9 women (BMI between 20 and $25 \mathrm{~kg} / \mathrm{m}^{2}$ ). There were no significant differences between the groups in age and height. The gender distribution was not statistically different between groups $\left(\chi^{2}=0.145, p=\right.$ 0.703). Demographic and anthropometric characteristics of both groups were shown in table 1.

The mean breathing frequencies of 38 subjects were $16.63 \pm 2.43$ breaths/min in the resting time and $15.83 \pm 3.75$ breaths/min during the metabolic measurements. No significant difference was found between the resting time breathing frequencies and breathing frequencies in the metabolic measurement time in all participants $(\mathrm{p}>0.05)$.

The mean RR was $911.79 \pm 88.60 \mathrm{~ms}$ in group 1 and $908.18 \pm 134.14 \mathrm{~ms}$ in group 2 . There was no statistically difference between these two groups $(\mathrm{p}>0.05)$. After $\log 10$ transformation was applied, the difference in total power between overweight/obese $\left(2,776.17 \pm 1,534.30 \mathrm{~ms}^{2}\right)$ and normal-weight subjects $\left(3,002.60 \pm 2,562.86 \mathrm{~ms}^{2}\right)$ was not significantly different $(\mathrm{p}>0.05)$. 
Fig. 1. The intercepts of the regression lines between REE and FFM.
Dal et al.: Resting Energy Expenditure in Normal-Weight and Overweight/Obese Subjects Was Similar Despite Elevated Sympathovagal Balance

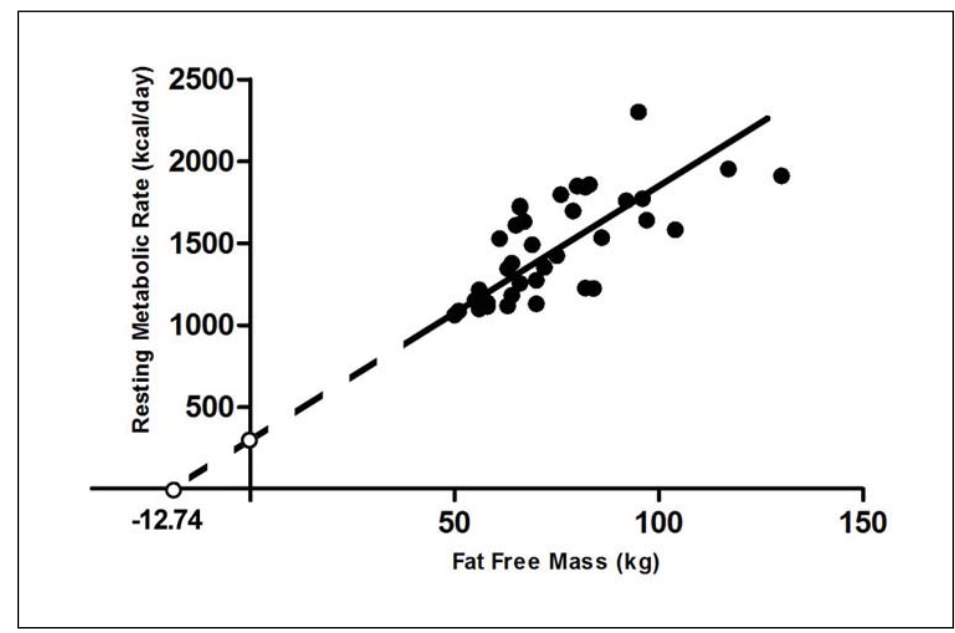

The mean LFnu was $61.11 \pm 17.09$ in group 1 and $44.51 \pm 18.63$ in group 2 . These values were significantly different $(\mathrm{p}<0.01)$. The mean HFnu was $38.89 \pm 17.09$ in group 1 and $55.50 \pm 18.63$ in group 2 , showing also a significant difference between the two groups $(\mathrm{p}<0.01)$.

The LF/HF ratio after $\log 10$ transformation was $2.32 \pm 2.09$ in group 1 and $1.10 \pm 1.05$ in group $2(\mathrm{p}<0.01)$.

Specifying REE data per kilogram, FFM is inappropriate to standardize the values of overweight/obese subjects and normal-weight subjects [17]. We calculated the intercepts of the regression line between REE and FFM for our study population by using multiple regression analysis. The regression formula was as follows: $\mathrm{REE}=274.37+21.74 \times \mathrm{FFM}$, where the straight regression line, when extended, cuts the $\mathrm{x}$-axis at the point $\mathrm{x}=-12.74$; this value was used as a correction number for FFM (fig. 1). Since the total number of subjects (38 subjects) who participated in the study was low, we divided REE by FFM plus $12.74 \mathrm{~kg}$ (FFMcorr) [16]. The mean FFMcorr was $74.55 \pm 12.50 \mathrm{~kg}$ in group 1 and $63.12 \pm 8.62 \mathrm{~kg}$ in group 2 , and there were statistically significant differences between the groups $(\mathrm{p}<0.05)$ after adding correction number to FFM.

The mean REE was $1,597.04 \pm 327.83 \mathrm{kcal} /$ day in group 1 while it was $1,367.76 \pm$ $264.35 \mathrm{kcal} /$ day in group 2 . The difference between two groups was statistically significant $(\mathrm{p}<0.05)$. REE was adjusted for corrected FFM $\left(\mathrm{REE}_{\mathrm{FFMcorr}}\right)$ according to the approach described above [16]. REE FFMcorr was $21.47 \pm 2.92 \mathrm{kcal} / \mathrm{day} / \mathrm{kg}$ in group 1 and $21.56 \pm 1.90$ $\mathrm{kcal} / \mathrm{day} / \mathrm{kg}$ in group 2; and this difference was not statistically significant ( $\mathrm{p}>0.05$ ). It was found that in group 1 FFMcorr explained $52.8 \%$ and fat mass $4.2 \%$ of the variation in REE while the respective values in group 2 were $80.6 \%$ and $6.5 \%$.

\section{Discussion}

Our study on spectral analysis of HRV demonstrated that although sympathovagal balance was elevated in overweight/obese subjects, REE adjusted for FFM was not different in overweight/obese and normal weight healthy subjects.

ANS has a vital role in the metabolic process related to the homeostatic regulation in the body. Cardiac autonomic activity can be assessed by spectral analysis of HRV. It was emphasized that the expression of LF and HF bands in normalized units reflects the relative 
balance between sympathetic and parasympathetic control, and the LF/HF ratio can be used as an index of sympathovagal balance [7]. In the literature, the hypothesis of 'an abnormality in ANS behavior in human obesity' is the point that all the studies agreed with, but there is a lack of agreement concerning the SNS activity in obesity. Several studies suggested reduced activity of SNS [10] or depressions both in sympathetic and parasympathetic activity [26]. The changes in the ANS activity result in impairment in metabolic processes that can lead to weight gain and vice versa [5, 27]. Insulin and leptin are the hormones that can affect the CNS to reduce food intake by appetite regulation and to increase energy expenditure by stimulating the sympathetic system. Activation of the renin-angiotensinaldosterone system is demonstrated in obesity before. Angiotensin can directly increase SNS activity [27]. It was reported that the balance between the sympathetic and parasympathetic nervous system can be altered in obese people [9]. The findings of our study showed that LFnu and LF/HF ratio increased and HFnu decreased in overweight/obese subjects compared to normal-weight subjects. These results may indicate an altered sympathovagal activity tending toward higher sympathetic activity and lower parasympathetic activity in overweight/obese subjects than in non-obese healthy subjects. In accordance with our hypothesis, Schmid et al. [9] and Lambert et al. [11] reported higher sympathetic activity in obese subjects.

REE in group 1 were statistically significantly higher than group 2 . The differences in REE between groups can be attributed to higher FFM in overweight/obese subjects [28]. However, for the comparison of REE between obese and non-obese subjects, it is recommended to use REE per unit of metabolically active mass because there is nonlinearity between REE and FFM. When REE measurements were expressed per kg FFM it can be overestimated in subjects with low FFM or underestimated in subjects with high FFM [17]. To overcome this situation, multiple regression analysis was used to determine the metabolically active body mass of the two groups $\left(\mathrm{FFM}_{\mathrm{corr}}\right)$. As a result of the analysis of REE adjusted for $\mathrm{FFM}_{\text {corr }}$, we found that there was no difference between overweight/obese and normalweight healthy subjects $(p>0.05)$. It was reported that REE decreases with weight loss and increases with weight gain $[27,29]$.

Elevated sympathovagal balance may lead to a higher REE level, but there was a close similarity of REE per kilogram of FFMcorr in overweight/obese and normal-weight healthy subjects in our study. Since our findings were determined by a cross-sectional study, there were no pre-obesity REE data of our subjects. REE might have been lower (like those in the normal-weight group) in group 1 in the pre-obesity period and gaining weight might have altered sympathovagal balance and induced REE to a new level. If the increment in REE in overweight/obese subjects had been higher than in non-obese subjects, it might have been thought that we could lose weight easily by dieting. Since REEFFMcorr was not high in overweight/obese subjects, additional methods such as exercise or calorie restriction are required to lose weight.

Based on the result of our study, FFMcorr explained $52.8 \%$ and fat mass $4.2 \%$ of the variation in REE while the respective values in group 2 were $80.6 \%$ and $6.5 \%$. In accordance with our results, it was shown that in obese individuals the contribution of FFM to REE was greater than that of fat mass [30,31]. The extracellular fluid (ECF) is also included in the FFM. Moreover, it was reported that adjusting FFM by ECF did not increase the ability of FFM to predict REE [30]. When the size of the metabolically active organ is increased, the active part of the tissue decreased; thus the metabolic activity of the larger FFM is lower than normal FFM [32]. Less metabolically active tissue may lead to lower contribution of FFM in the group 1.

One limitation of this study was that we were not able to analyze ANS activity by other methods, e.g., urinary catecholamine excretion and/or neural conductivity which may lead 
to more accurate assessment of the SNS activity. Longitudinal studies are required to assess the REE and HRV interaction in the weight gaining process. In our study, the subjects in the overweight/obese group were rather young and did not suffer from any illness resulting from overweight. We accepted this period as a time where weight loss must begin to protect against the obesity-induced illness and death [33-35] and we advised the participants to join in a weight loss program in a hospital. It was also demonstrated that ANS activity and REE changed after weight loss [3,29].

\section{Conclusions}

To avoid misestimation of the REE in overweight/obese and normal-weight subjects, the REE should be adjusted for corrected FFM. Contrary to our expectations, the findings of the present study indicated that $\mathrm{REE}_{\mathrm{FFMcor}}$ levels in overweight/obese and non-obese subjects were not different despite elevated sympathovagal balance in overweight/obese individuals.

\section{Acknowledgements}

The authors wish to thank to Bora Resitoglu and Figen Dag for their support in conducting this study.

\section{Disclosure Statement}

No conflict of interest was declared by the authors.

\section{References}

1 World Health Organization: Obesity: preventing and managing the global epidemic. Report of a WHO consultation. World Health Organization Technical Report Series 2000;894:i-xii,1-253.

- 2 Beckers S, Zegers D, Van Gaal LF, Van Hul W: Replication of the SH2B1 rs7498665 association with obesity in a Belgian study population. Obes Facts 2011;4:473-477.

3 Reinehr T: Obesity and thyroid function. Mol Cell Endocrinol 2010;316:165-171.

- 4 Weaver J: Classical endocrine diseases causing obesity. Front Horm Res 2008;36:212-228.

- 5 Kimura T, Matsumoto T, Akiyoshi M, Owa Y, Miyasaka N, Aso T, Moritani T: Body fat and blood lipids in postmenopausal women are related to resting autonomic nervous system activity. Eur J Appl Physiol 2006;97: 542-547.

6 Task Force of The European Society of Cardiology and The North American Society of Pacing and Electrophysiology: Heart rate variability: standards of measurement, physiological interpretation, and clinical use. Eur Heart J 1996;17: 354-381.

- 7 Berntson GG, Bigger JT, Eckberg DL, Grossman P, Kaufmann PG, Malik M, Nagaraja HN, Porges SW, Saul JP, Stone PH, van der Molen MW: Heart rate variability: origins, methods, and interpretive caveats. Psychophysiology 1997;34:623-648.

- 8 Perini R, Veicsteinas A: Heart rate variability and autonomic activity at rest and during exercise in various physiological conditions. Eur J Appl Physiol 2003;90(:317-325.

- 9 Schmid K, Schönlebe J, Drexler H, Mueck-Weymann M: Associations between being overweight, variability in heart rate, and well-being in the young men. Cardiol Young 2010;20:54-59.

10 Bray GA: Obesity, a disorder of nutrient partitioning: the MONA LISA hypothesis. J Nutr 1991;121:11461162 .

11 Lambert GW, Straznicky NE, Lambert EA, Dixon JB, Schlaich MP: Sympathetic nervous activation in obesity and the metabolic syndrome - causes, consequences and therapeutic implications. Pharmacol Ther 2010; 126:159-172.

12 Haugen HA, Chan LN, Li F: Indirect calorimetry: a practical guide for clinicians. Nutr Clin Pract 2007;22: 377-388. 
13 Wilms B, Schmid SM, Ernst B, Thurnheer M, Mueller MJ, Schultes B: Poor prediction of resting energy expenditure in obese women by established equations. Metabolism 2010;59:1181-1189.

14 Schaefer F, Georgi M, Zieger A, Schärer K: Usefulness of bioelectric impedance and skinfold measurements in predicting fat-free mass derived from total body potassium in children. Pediatr Res 1994;35:617-624.

15 Eston RG, Fu F, Fung L: Validity of conventional anthropometric techniques for predicting body composition in healthy Chinese adults. Br J Sport Med 1995;29:52-56.

16 Lukaski HC: Methods for the assessment of human-body composition - traditional and new. Am J Clin Nutr 1987:46:537-556.

17 Ravussin E, Bogardus C: Relationship of genetics, age, and physical fitness to daily energy expenditure and fuel utilization. Am J Clin Nutr 1989;49:968-975.

18 Schwartz MH: Protocol changes can improve the reliability of oxygen cost data. Gait Posture 2007;26:494500.

19 Matarese LE: Indirect calorimetry: technical aspects. J Am Diet Assoc 1997;97:154-160.

20 Lowe JC, Yellin J, Honeyman-Lowe G: Female fibromyalgia patients: lower resting metabolic rates than matched healthy controls. Med Sci Monit 2006;12:CR282-CR289.

21 Gamelin FX, Berthoin S, Bosquet L: Validity of the Polar S810 heart rate monitor to measure R-R intervals at rest. Med Sci Sports Exerc 2006;38:887-893.

22 Vanderlei LCM, Silva RA, Pastre CM, Azevedo FM, Godoy MF: Comparison of the Polar S810i monitor and the ECG for the analysis of heart rate variability in the time and frequency domains. Braz J Med Biol Res 2008;41:854-859.

23 Nunan D, Jakovljevic DG, Donovan G, Hodges LD, Sandercock GRH, Brodie DA: Levels of agreement for RR intervals and short-term heart rate variability obtained from the Polar S810 and an alternative system. Eur J Appl Physiol 2008;103:529-537.

-24 Pichon A, Roulaud M, Antoine-Jonville S, de Bisschop C, Denjean A: Spectral analysis of heart rate variability: interchangeability between autoregressive analysis and fast Fourier transform. J Electrocardiol 2006;39:31-37.

-25 Gamelin FX, Baquet G, Berthoin S, Thevenet D, Nourry C, Nottin S, Bosquet L: Effect of high intensity intermittent training on heart rate variability in prepubescent children. Eur J Appl Physiol 2009;105:731-738.

-26 Laederach-Hofmann K, Mussgay L, Rúddel H: Autonomic cardiovascular regulation in obesity. J Endocrinol 2000;164: 59-66.

27 Davy KP, Orr JS: Sympathetic nervous system behavior in human obesity. Neurosci Biobehav Rev 2009;33: 116-124.

-28 Shibao C, Gamboa A, Diedrich A, Ertl AC, Chen KY, Byrne DW, Farley G, Paranjape SY, Davis SN, Biaggioni I: Autonomic contribution to blood pressure and metabolism in obesity. Hypertension 2007;49:27-33.

29 Leibel RL, Rosenbaum M, Hirsch J: Changes in energy expenditure resulting from altered body weight. N Engl J Med 1995;332:621-628.

-30 Nielsen S, Hensrud DD, Romanski S, Levine JA, Burguera B, Jensen MD: Body composition and resting energy expenditure in humans: role of fat, fat-free mass and extracellular fluid. Int J Obes Relat Metab Disord 2000;24:1153-1157.

-31 Cunningham JJ: Body composition as a determinant of energy expenditure: a synthetic review and a proposed general prediction equation. Am J Clin Nutr 1991;54:963-969.

-32 Weinsier RL, Schutz Y, Bracco D: Reexamination of the relationship of resting metabolic rate to fat-free mass and to the metabolically active components of fat-free mass in humans. Am J Clin Nutr 1992;55:790794.

-33 Duncan M, Griffith M, Rutter H, Goldacre MJ: Certification of obesity as a cause of death in England 19792006. Eur J Public Health 2010;20:671-675.

-34 Klein S, Sheard NF, Pi-Sunyer X, Daly A, Wylie-Rosett J, Kulkarni K, Clark NG: Weight management through lifestyle modification for the prevention and management of type 2 diabetes: rationale and strategies. A statement of the American Diabetes Association, the North American Association for the Study of Obesity, and the American Society for Clinical Nutrition. Diabetes Care 2004;27:2067-2073.

35 Marinou K, Tousoulis D, Antonopoulos AS, Stefanadi E, Stefanadis C: Obesity and cardiovascular disease: from pathophysiology to risk stratification. Int J Cardiol 2010;138:3-8. 